\title{
The impact of the skim milk powder manufacturing process on the flavor of model white chocolate
}

Article

Accepted Version

Stewart, A., Grandison, A. S., Ryan, A., Festring, D., Methven, L. and Parker, J. K. (2017) The impact of the skim milk powder manufacturing process on the flavor of model white chocolate. Journal of Agricultural and Food Chemistry, 65 (6). pp. 11861195. ISSN 0021-8561 doi:

https://doi.org/10.1021/acs.jafc.6b04489 Available at https://centaur.reading.ac.uk/68637/

It is advisable to refer to the publisher's version if you intend to cite from the work. See Guidance on citing.

To link to this article DOI: http://dx.doi.org/10.1021/acs.jafc.6b04489

Publisher: American Chemical Society

All outputs in CentAUR are protected by Intellectual Property Rights law, including copyright law. Copyright and IPR is retained by the creators or other copyright holders. Terms and conditions for use of this material are defined in the End User Agreement. 


\section{CentAUR}

Central Archive at the University of Reading

Reading's research outputs online 
The Impact of the Skim Milk Powder Manufacturing Process on the Flavor of Model White Chocolate

Ashleigh Stewart, ${ }^{\dagger}$ Alistair S. Grandison, ${ }^{\dagger}$ Angela Ryan, ${ }^{\S}$ Daniel Festring,${ }^{\S}$ Lisa Methven, ${ }^{\dagger}$ Jane K. Parker ${ }^{\dagger}, *$

$\dagger$ Department of Food and Nutritional Sciences, University of Reading, Reading RG6 6AP, UK

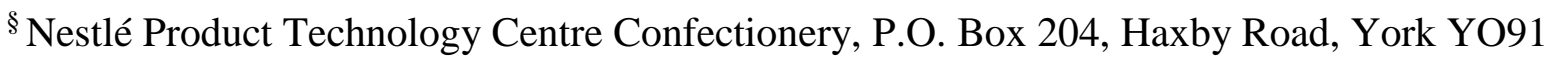
1XY, UK

*Corresponding Author:

Tel: +44 1183787455

E-mail: j.k.parker@ reading.ac.uk 
Table S1 Reference materials provided to help assessors to standardize attribute descriptors

Descriptor Reference material

\begin{tabular}{ll}
\hline sour cream, lactic, cheesy (odor and flavor) & natural yogurt \\
cocoa butter (odor) & cocoa butter hand cream \\
caramel (odor) & caramel syrup \\
brown sugar (flavor) & muscovado sugar \\
caramel (flavor) & caramel syrup \\
fudge (flavor) & dairy fudge* \\
condensed milk (odor and flavor) & evaporated and sweetened \\
& condensed-milk \\
creamy (flavor) & cream \\
nutty (odor and flavor) & roasted hazelnuts
\end{tabular}

* Dairy fudge purchased from a UK supermarket was a typical example of a UK fudge, consisting of only sugar, butter and condensed skim milk 
Table S2 Mean panel scores $(n=9)$ for sensory attributes of two types of white chocolate produced using skim milk powders of different heat treatments - high heat (HHCHOC), low heat (LHCHOC)

\begin{tabular}{|c|c|c|c|c|c|c|c|}
\hline \multirow[t]{2}{*}{ attribute } & & \multicolumn{2}{|c|}{$\underset{a}{\text { score }}$} & \multirow{2}{*}{$\underset{b}{\mathrm{LSD}}$} & \multicolumn{3}{|c|}{$\mathrm{P}$} \\
\hline & & $\mathrm{HH}$ CHOC & LH CHOC & & S & A & I \\
\hline \multicolumn{8}{|c|}{ appearance } \\
\hline & shininess & 17 & 18 & 9.0 & $\mathrm{~ns}$ & $*$ & ns \\
\hline & yellow & 54 & 34 & 3.5 & $* * *$ & $* * *$ & ns \\
\hline \multicolumn{8}{|l|}{ odor } \\
\hline & sweet & 37 & 38 & 7.0 & $\mathrm{~ns}$ & $* *$ & ns \\
\hline & vanilla & 16 & 16 & 6.9 & $\mathrm{~ns}$ & $*$ & $\mathrm{~ns}$ \\
\hline & caramel & 22 & 13 & 12 & $\mathrm{~ns}$ & ns & $* * *$ \\
\hline & evaporated milk & 32 & 24 & 12 & $\mathrm{~ns}$ & ns & ns \\
\hline & cheesy & 6.4 & 4.5 & 6.5 & $\mathrm{~ns}$ & $\mathrm{~ns}$ & $*$ \\
\hline & cocoa butter & 16 & 12 & 7.5 & $\mathrm{~ns}$ & $*$ & $*$ \\
\hline & cardboard & 8.9 & 7.6 & 7.0 & $\mathrm{~ns}$ & $* *$ & $* *$ \\
\hline \multicolumn{8}{|l|}{ taste } \\
\hline & sweet & 48 & 47 & 7.2 & $\mathrm{~ns}$ & $* *$ & ns \\
\hline & acidic & 7.8 & 6.9 & 3.4 & $\mathrm{~ns}$ & $* *$ & ns \\
\hline & salty & 7.0 & 7.3 & 1.3 & $\mathrm{~ns}$ & $* * *$ & ns \\
\hline \multicolumn{8}{|l|}{ flavor } \\
\hline & overall flavor intensity & 53 & 43 & 5.5 & $* *$ & $*$ & ns \\
\hline & vanilla & 18 & 18 & 3.1 & $\mathrm{~ns}$ & $* * *$ & ns \\
\hline & fudge & 28 & 16 & 11.9 & $*$ & ns & $* * *$ \\
\hline & condensed-milk & 31 & 23 & 6.3 & $*$ & ns & ns \\
\hline & cheesy & 4.1 & 2.2 & 5.3 & $\mathrm{~ns}$ & ns & $* *$ \\
\hline & cocoa butter & 15 & 12 & 6.5 & $\mathrm{~ns}$ & $*$ & ns \\
\hline \multicolumn{8}{|c|}{ mouthfeel } \\
\hline & hardness of bite & 29 & 42 & 11 & $*$ & ns & $*$ \\
\hline & speed of melting & 38 & 33 & 11 & $\mathrm{~ns}$ & $*$ & $* * *$ \\
\hline & mouth coating & 31 & 33 & 8.2 & $\mathrm{~ns}$ & $* *$ & $\mathrm{~ns}$ \\
\hline & smoothness & 57 & 59 & 6.8 & $\mathrm{~ns}$ & $* * *$ & ns \\
\hline & grains & 6.3 & 5.2 & 4.7 & $\mathrm{~ns}$ & $*$ & $*$ \\
\hline & mouth-watering & 22 & 21 & 4.0 & $\mathrm{~ns}$ & $* * *$ & ns \\
\hline & fatty & 28 & 32 & 10 & $\mathrm{~ns}$ & $*$ & $\mathrm{~ns}$ \\
\hline & adhesive & 27 & 26 & 10 & $\mathrm{~ns}$ & * & $\mathrm{ns}$ \\
\hline & mouth drying & 13 & 13 & 5.4 & $\mathrm{~ns}$ & $* *$ & $*$ \\
\hline & tongue tingling & 1.3 & 1.4 & 3.6 & $\mathrm{~ns}$ & ns & $* *$ \\
\hline & throat catching & 7.0 & 11 & 8.5 & $\mathrm{~ns}$ & ns & $*$ \\
\hline \multicolumn{8}{|c|}{ after-effects } \\
\hline & sweet & 41 & 40 & 6.2 & $\mathrm{~ns}$ & $* *$ & ns \\
\hline & salt & 5.3 & 5.3 & 2.0 & $\mathrm{~ns}$ & $* * *$ & ns \\
\hline & acidic & 6.7 & 5.7 & 3.2 & $\mathrm{~ns}$ & $* *$ & ns \\
\hline & mouth drying & 14 & 12 & 3.0 & $\mathrm{~ns}$ & $* * *$ & ns \\
\hline & mouth coating after swallow & 19 & 16 & 7.0 & $\mathrm{~ns}$ & $*$ & $*$ \\
\hline
\end{tabular}

\footnotetext{
${ }^{a}$ Means not labelled with the same letters are significantly different $(\mathrm{p}<0.05)$; means of two replicate assessment for each assessor (18 replicates in total).

${ }^{\mathrm{b}}$ Least significance difference at $\mathrm{p}=0.05$.

${ }^{c}$ Probability, obtained from ANOVA, that there is a difference between means; ns, no significant difference between means (p>0.05); * significant at the 5\% level; ** significant at the $1 \%$ level; *** significant at the $0.1 \%$ level; F-ratios for sample and assessor were calculated by comparing the mean square of the effect with the mean square of the sample $\times$ assessor interaction; S: significance of samples, A: significance of assessors, I: significance of the interaction $(\mathrm{S} \times \mathrm{A})$.
} 